\title{
Crk-like adapter protein is overexpressed in cervical carcinoma, facilitates proliferation, invasion and chemoresistance, and regulates Src and Akt signaling
}

\author{
HONG JI ${ }^{1,2}$, BO LI ${ }^{1}$, SHITAI ZHANG ${ }^{1}$, ZHENG HE $^{1}$, YANG ZHOU ${ }^{1}$ and LING OUYANG ${ }^{1}$ \\ ${ }^{1}$ Department of Gynecology and Obstetrics, Shengjing Hospital of China Medical University, Shenyang, Liaoning 110004; \\ ${ }^{2}$ Department of Gynecology and Obstetrics, First Affiliated Hospital of Jinzhou Medical University, \\ Jinzhou, Liaoning 121000, P.R. China
}

Received April 6, 2015; Accepted July 1, 2016

DOI: $10.3892 / 01.2016 .5160$

\begin{abstract}
Overexpression of Crk-like (CrkL) adapter protein has been implicated in a number of types of human cancer. However, its involvement in human cervical carcinoma remains unclear. The present study aimed to explore the clinical significance and biological characteristics of CrkL in human cervical carcinoma. CrkL protein expression was examined in tissue samples from 92 cases of cervical carcinoma using immunohistochemistry, and was found to be overexpressed in $48.9 \%$ (45/92 cases). CrkL was transfected into HeLa and CaSki cervical carcinoma cell lines and its effects on biological behavior were examined. CrkL overexpression was revealed to promote cell proliferation, invasion and chemoresistance. In addition, CrkL overexpression increased the level of Src and Akt phosphorylation. Treatment with the Src inhibitor dasatinib eliminated the effect of CrkL on cell invasion. In conclusion, the current results demonstrate that CrkL is an oncoprotein overexpressed in cervical carcinoma which contributes to malignant cell growth and chemoresistance. In addition, the findings indicate that $\mathrm{CrkL}$ promotes cervical cancer cell invasion through a Src-dependent pathway.
\end{abstract}

\section{Introduction}

Cervical cancer is one of most common gynecological malignancies (1) and the incidence is increasing in China, where the age-specific incidence rate increased from 8.76 to 23.1 cases per 100,000 individuals between 1993 and 2008 (2). Despite the treatment of cervical cancer patients with surgery and

Correspondence to: Professor Ling Ouyang, Department of Gynecology and Obstetrics, Shengjing Hospital of China Medical University, 36 Sanhao Street, Heping, Shenyang, Liaoning 110004, P.R. China

E-mail: ouyanglingcmu@163.com

Key words: Crk-like, cervical carcinoma, invasion, Src adjuvant therapy, such as radiotherapy and chemotherapy, the effectiveness of treatment has not improved significantly over the past decades $(3,4)$. In China, annual incidence and mortality rates have increased from 10.4 cases and 1.22 mortalities per 100,000 individuals, respectively, to 13.4 cases and 2.59 mortalities per 100,000 individuals between 2003 and 2011 (5). Thus, it is important to identify molecular markers that are able to predict the malignant phenotype of cervical carcinoma $(6,7)$.

Crk-like (CrkL) adapter protein has been reported to be involved in numerous biological activities, such as cell proliferation and migration, and plays an important role in leukemia (8-11). CrkL proteins contain two Src homology (SH) 3 domains and one N-terminal SH2 domain, which could bind various docking proteins, including p130Cas, paxillin and Bcr-Abl $(9,12,13)$. Recently, CrkL protein has been demonstrated to be overexpressed in a number of types of human cancer, and to induce cancer cell proliferation and invasion (14-18). Overexpression of CrkL in fibroblast cells promotes anchorage-independent growth (19). Additionally, activating mutations of anaplastic lymphoma kinase have been shown to exert this protein's downstream effects through CrkL (20). Collectively, these findings implicate CrkL as an important oncoprotein in human cancers. However, the expression pattern and biological roles of CrkL in cervical carcinoma remain unexplored.

In the present study, CrkL protein expression was examined in specimens from 92 cases of cervical carcinoma. In addition, CrkL expression was upregulated in HeLa and CaSki cell lines and its effect on cell proliferation and apoptosis assessed. Furthermore, the molecular signaling pathways underlying the biological effects of CrkL were investigated.

\section{Materials and methods}

Patients and specimens. The study protocol was approved by the Institutional Review Board of Shengjing Hospital of China Medical University (Shenyang, China). Primary tumor specimens were obtained from patients diagnosed with cervical carcinoma who underwent resection at the First Affiliated Hospital of Jinzhou Medical University (Jinzhou, 
China) and Shengjing Hospital of China Medical University between January 2009 and December 2012. Normal endocervical tissues were obtained from patients with benign uterine disease without cervical dysplasia. The histological diagnosis was evaluated in sections stained with hematoxylin and eosin, according to the World Health Organization classification guidelines. Clinical and histopathological data were obtained from medical records.

Immunohistochemistry. Surgically excised tumor specimens were fixed with $10 \%$ neutral formalin and embedded in paraffin, and $4 \mu \mathrm{m}$-thick sections were prepared. Immunostaining was performed using the Elivision Plus Polyer HRP (Mouse/Rabbit) IHC kit (Fuzhou Maixin Biotech. Co., Ltd., Fuzhou, China). The sections were deparaffinized in xylene, rehydrated with graded alcohol and then boiled in $0.01 \mathrm{M}$ citrate buffer ( $\mathrm{pH}$ 6.0) for $2 \mathrm{~min}$ in an autoclave. Hydrogen peroxide $(0.3 \%)$ was applied to block endogenous peroxide activity and the sections were incubated with normal goat serum to reduce nonspecific binding. Tissue sections were incubated with an anti-CrkL rabbit polyclonal antibody (dilution, 1:600; cat. no. ABC242; EMD Millipore, Billerica, MA, USA;) at $4^{\circ} \mathrm{C}$ overnight. A biotinylated goat anti-rabbit horseradish peroxidase polymer (dilution, 1:800; cat. no. KIT-9902B; Fuzhou Maixin Biotech. Co., Ltd.) was used as a secondary antibody at room temperature for $30 \mathrm{~min}$. After washing, the peroxidase reaction was developed with DAB. Counterstaining with hematoxylin was performed and the sections were dehydrated in ethanol prior to mounting.

Two independent investigators, who were blinded to the patient characteristics, examined all tumor slides randomly: Five views were examined per slide, and 100 cells were observed per view at $\mathrm{x} 400$ magnification. In accordance with previous reports, immunostaining of CrkL was scored on a semi-quantitative scale by evaluating the intensity and percentage of tumor cells $(18,21)$. Cytoplasmic and membrane immunostaining was considered positive staining. After counting 400 tumor cells, the percentage of positively stained cells was calculated. The intensity of CrkL staining was scored as 0 (no signal), 1 (moderate) or 2 (strong). Percentage scores were assigned as $1(1-25 \%), 2(26-50 \%), 3(51-75 \%)$ or 4 (76-100\%). The scores of each tumor sample were multiplied to give a final score of $0-8$; tumor samples that scored $4-8$ were considered to exhibit CrkL overexpression.

Cell culture and transfection. HeLa and CaSki cell lines were obtained from the American Type Culture Collection (Manassas, VA, USA). Cells were cultured in Invitrogen Dulbecco's modified Eagle's medium (DMEM; Thermo Fisher Scientific, Inc., Carlsbad, CA, USA) containing $10 \%$ fetal calf serum (FCS) (Invitrogen; Thermo Fisher Scientific, Inc.), $100 \mathrm{IU} / \mathrm{ml}$ penicillin (Sigma-Aldrich, St. Louis, MO, USA), and $100 \mu \mathrm{g} / \mathrm{ml}$ streptomycin (Sigma-Aldrich). Cells were grown in sterilized culture dishes and were passaged every 2 days with $0.25 \%$ trypsin.

The plasmid pCMV6-CrkL was purchased from OriGene Technologies, Inc. (Rockville, MD, USA). Plasmid was transfected into cells using Lipofectamine LTX reagent (Invitrogen; Thermo Fisher Scientific, Inc.). pCMV6 empty vector (pCMV6 EV) was used as a negative control. Cisplatin
(Santa Cruz Biotechnology, Inc., Santa Cruz, CA, USA). was dissolved in dimethyl sulfoxide (DMSO) and $10 \mu \mathrm{M}$ cisplatin was used to treat cancer cells for $24 \mathrm{~h}$. DMSO was used as the negative control.

Reverse transcription-quantitative polymerase chain reaction (RT-qPCR) using SYBR Green method. Total RNA was extracted from HeLa and CaSki cells using Trizol (Life Technologies; Thermo Fisher Scientific, Inc.) according to the manufacturer's instructions. RNA was reverse transcribed into cDNA using PrimerScript RT Master Mix kit (Takara Bio, Dalian, China) at $85^{\circ} \mathrm{C}$ for $2 \mathrm{~min}$ and $37^{\circ} \mathrm{C}$ for $30 \mathrm{~min}$. Briefly, $20 \mu 1$ reverse-transcription reaction solution was prepared using 5X RT Master Mix (4 $\mu \mathrm{l})$ and $800 \mathrm{ng}$ RNA. RT-qPCR was performed using SYBR Select PCR Master Mix (Applied Biosystems; Thermo Fisher Scientific, Inc.) in a total volume of $20 \mu \mathrm{l}$ on an Applied Biosystems 7300 Real-Time PCR System (Thermo Fisher Scientific, Inc.), with the following conditions: $95^{\circ} \mathrm{C}$ for $30 \mathrm{sec}$; and 40 cycles of $95^{\circ} \mathrm{C}$ for $5 \mathrm{sec}$ and $60^{\circ} \mathrm{C}$ for $30 \mathrm{sec}$. The PCR solution $(20 \mu \mathrm{l})$ consisted of $5 \mu \mathrm{l}$ cDNA, $0.5 \mu \mathrm{l}$ forward primer, $0.5 \mu \mathrm{l}$ reverse primer, $4 \mu \mathrm{l} ; \mathrm{H}_{2} \mathrm{O}$ and $10 \mu \mathrm{l}$ SYBRgreen Master mix (Applied Biosystems; Thermo Fisher Scientific, Inc.). A dissociation step was performed to generate a melting curve to confirm the specificity of the amplification. $\beta$-actin was used as the reference gene. The relative levels of gene expression were calculated by the $2^{-\Delta \Delta \mathrm{Cq}}$ method (22). The primer sequences were as follows: CrkL forward, 5'-CCTTTGCCATCCACA CAGAAT-3', CrkL reverse, 5'-TTTCACGATGTCACCAAC CTCTA-3'; $\beta$-actin forward, 5'-ATAGCACAGCCTGGATAG CAACGTAC- 3 ', and $\beta$-actin reverse, 5'-CACCTTCTACAA TGAGCTGCGTGTG-3'. All experiments were performed in triplicate.

Western blot analysis. Total proteins from HeLa and CaSki cells were extracted and quantified using the Bradford method, and $20 \mathrm{mg}$ protein was separated by SDS-PAGE. Samples were transferred to polyvinylidene difluoride membranes (EMD Millipore) and incubated overnight at $4^{\circ} \mathrm{C}$ with antibodies against CrkL (rabbit polyclonal; dilution, 1:1,000; cat. no. ABC242; EMD Millipore), p-Src (rabbit monoclonal; dilution, 1:1,000; cat. no. 12432; Cell Signaling Technology, Inc., Boston, MA, USA), Src (rabbit monoclonal; dilution, 1:1,000; cat. no. 2109; Cell Signaling Technology, Inc.), p-Akt (rabbit polyclonal; dilution, 1:1,000; cat. no. 9271; Cell Signaling Technology, Inc.), Akt (rabbit polyclonal; dilution, 1:1,000; cat. no. 9272; Cell Signaling Technology, Inc.; dilution, 1:1,000) and GAPDH (rabbit polyclonal; dilution, 1:1,000; cat. no. G5262; Santa Cruz Biotechnology, Inc.). Following incubation with peroxidase-coupled anti-mouse/rabbit IgG (dilution, 1:2,000; cat. no. 5127; Cell Signaling Technology, Inc.) at $37^{\circ} \mathrm{C}$ for $2 \mathrm{~h}$, proteins were visualized using Pierce ECL Western Blotting Substrate (Thermo Fisher Scientific, Inc.) and detected using a DNR Bio-Imaging System (DNR Bio-Imaging Systems Ltd., Jerusalem, Israel).

Methyl thiazolyl tetrazolium (MTT) assay. For the MTT cell viability assay, at $24 \mathrm{~h}$ after transfection, cells were plated in 96-well plates at a concentration of $\sim 2,000$ cells/well 
Table I. Distribution of CrkL status in cervical carcinoma according to clinicopathological characteristics.

\begin{tabular}{|c|c|c|c|c|}
\hline \multirow[b]{2}{*}{ Characteristic } & \multirow{2}{*}{$\begin{array}{c}\text { Total } \\
\text { patients, } \mathrm{n}\end{array}$} & \multicolumn{2}{|c|}{ CrkL status, $\mathrm{n}$} & \multirow[b]{2}{*}{ P-value } \\
\hline & & Weak/negative & Positive & \\
\hline Age, years & & & & 0.2106 \\
\hline$<50$ & 61 & 34 & 27 & \\
\hline$\geq 50$ & 31 & 13 & 18 & \\
\hline Histological type & & & & 0.9419 \\
\hline Squamous cell carcinoma & 82 & 42 & 40 & \\
\hline Adenocarcinoma & 10 & 5 & 5 & \\
\hline Differentiation & & & & 0.5647 \\
\hline Well/moderate & 67 & 33 & 34 & \\
\hline Poor & 25 & 14 & 11 & \\
\hline TNM stage & & & & 0.0165 \\
\hline I & 36 & 24 & 12 & \\
\hline II+III & 56 & 23 & 33 & \\
\hline T stage & & & & 0.0972 \\
\hline $\mathrm{T} 1$ & 49 & 29 & 20 & \\
\hline $\mathrm{T} 2+3$ & 43 & 18 & 25 & \\
\hline Lymph node metastasis & & & & 0.0212 \\
\hline Negative & 56 & 34 & 22 & \\
\hline Positive & 36 & 13 & 23 & \\
\hline
\end{tabular}

CrkL, Crk-like; TNM, tumor-node-metastasis.
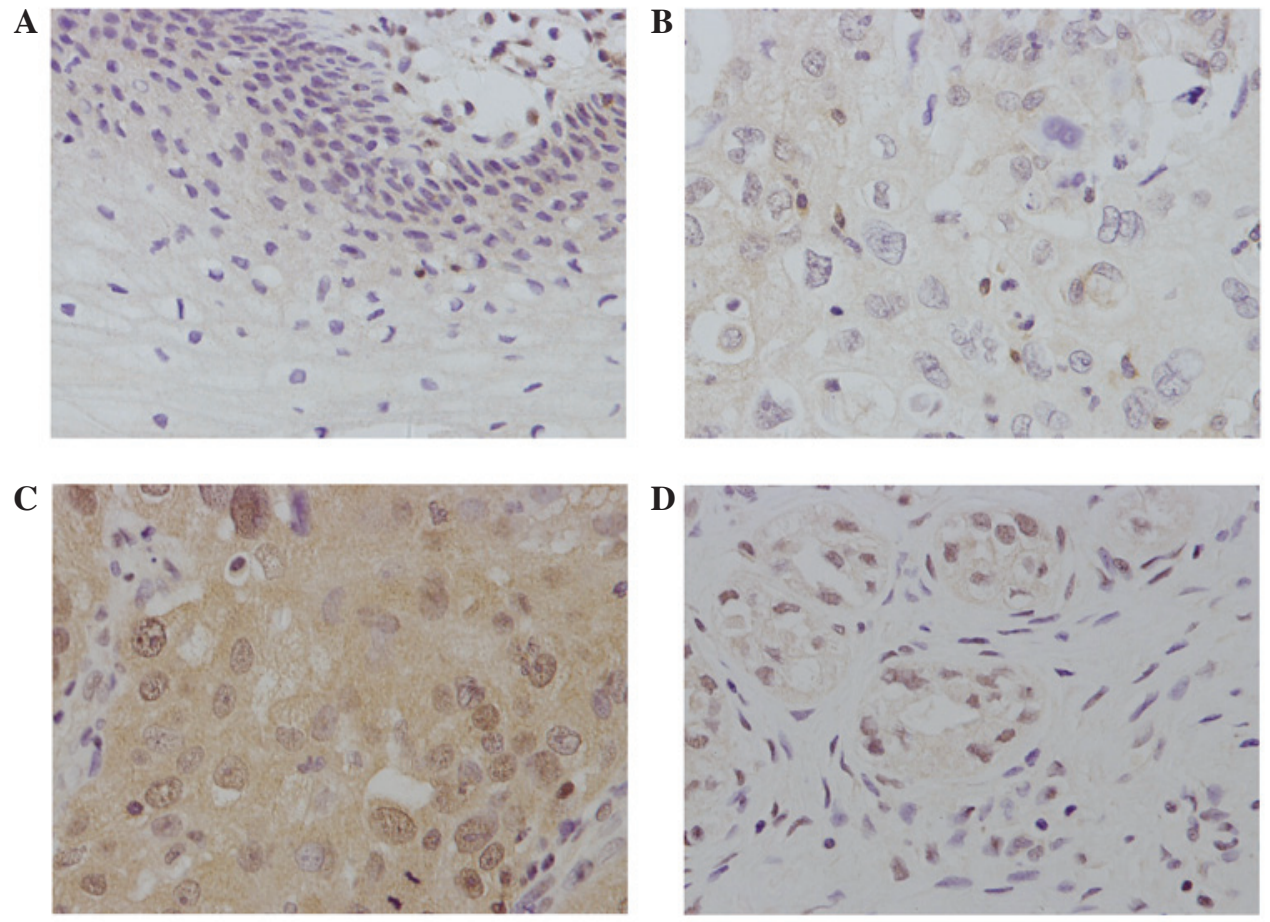

Figure 1. Expression of CrkL protein in cervical carcinoma tissues. (A) Negative CrkL expression in normal cervical tissue. (B) Weak CrkL expression in a case of stage II carcinoma. (C) Positive staining of CrkL in a case of stage III cervical carcinoma. (D) Negative CrkL expression in a case of stage I adenocarcinoma (magnification, x400). Brown cytoplasmic and membrane staining was considered positive. CrkL, Crk-like.

and cultured for 5 days. To quantitate cell viability, $20 \mu 1$ of $5 \mathrm{mg} / \mathrm{ml}$ MTT (thiazolyl blue) solution was added to each well and incubated for $4 \mathrm{~h}$ at $37^{\circ} \mathrm{C}$. The medium was removed from each well and the resulting MTT formazan 
A

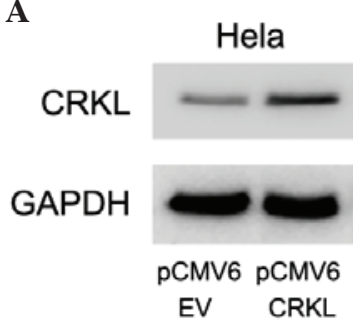

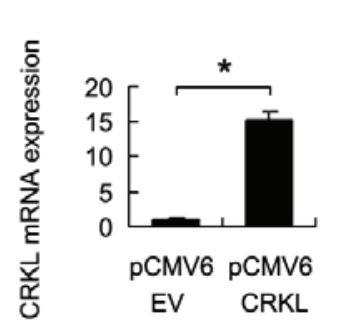

B

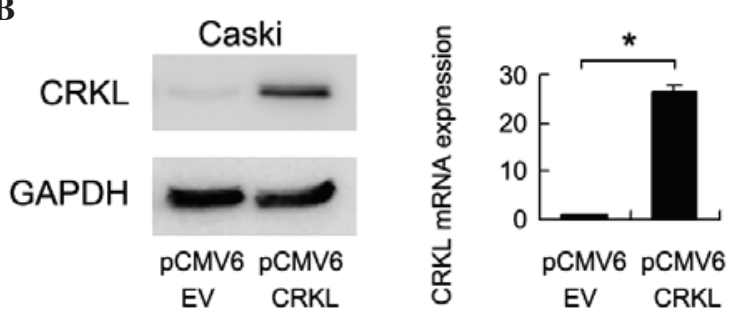

Figure 2. Transfection of CrkL plasmid (pCMV6 CrkL) upregulates endogenous CrkL expression in cervical carcinoma cell lines. Western blot and quantitative polymerase chain reaction analysis revealed that CrkL transfection in (A) HeLa and (B) Caski cell lines upregulated its protein expression. ${ }^{*} \mathrm{P}<0.05$. CrkL, Crk-like; GAPDH, glyceraldehyde 3-phosphate dehydrogenase; pCMV6 EV, empty vector.

A

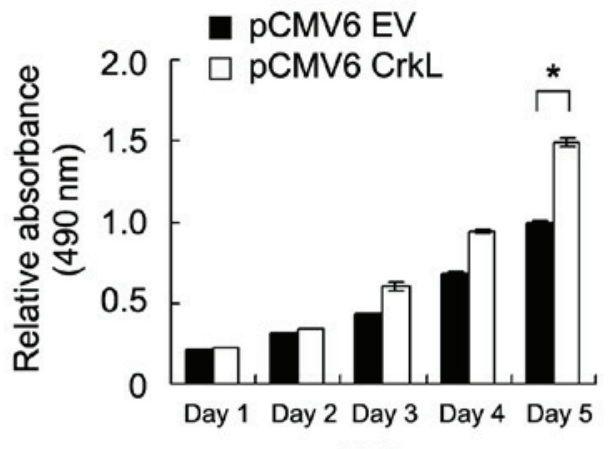

HeLa
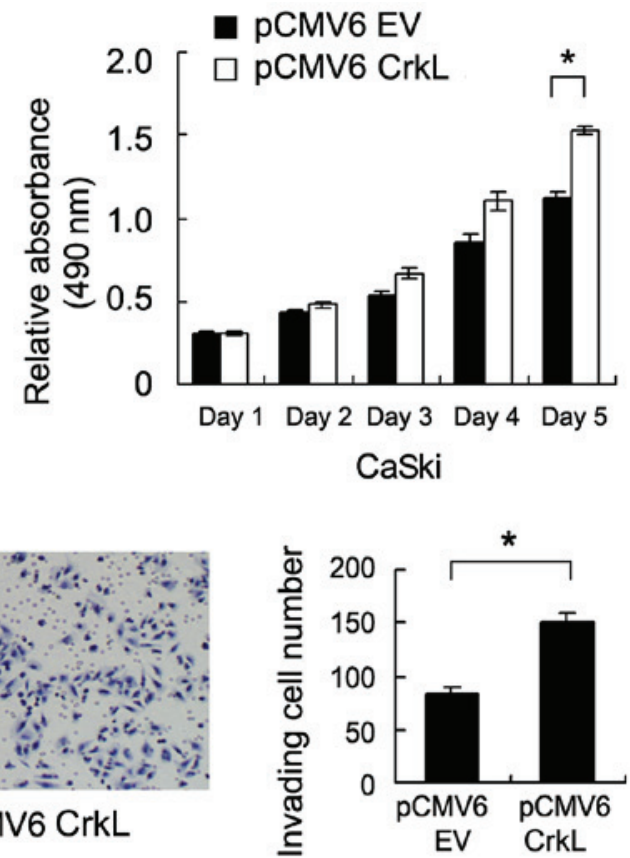

pCMV6 CrkL

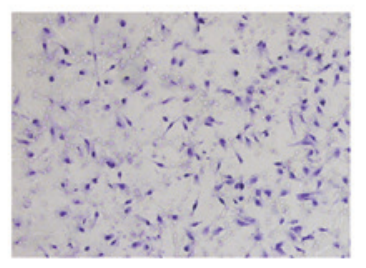

pCMV6 CrkL

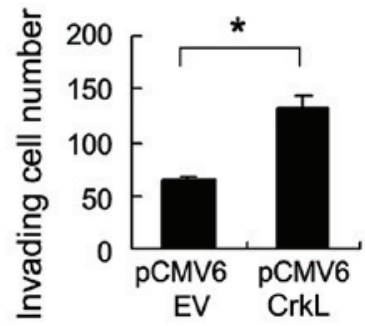

pCMV6 EV $\square$ pCMV6 CrkL

C
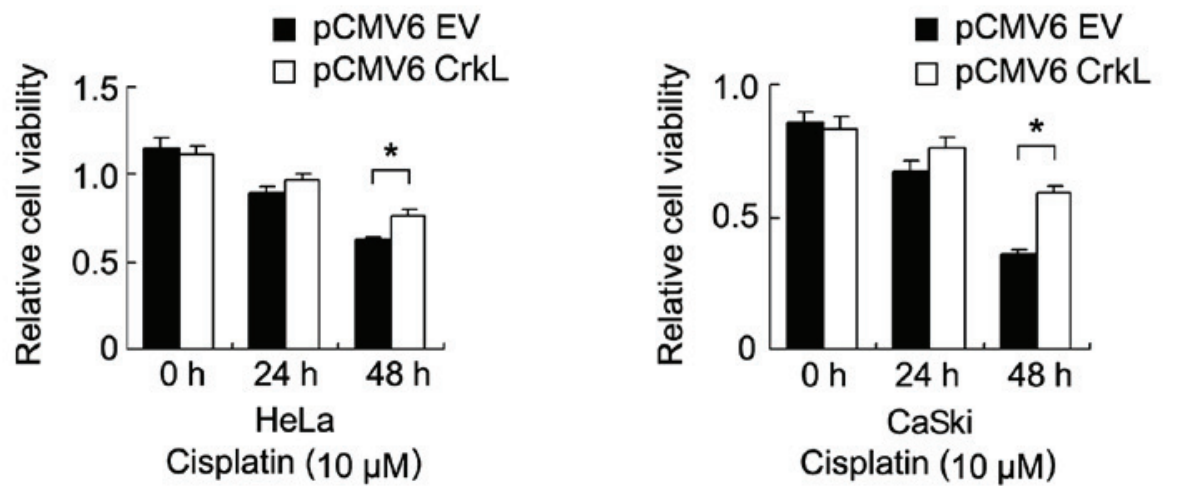

Figure 3. CrkL promotes proliferation, invasion and chemoresistance of HeLa and CaSki cells. (A) An MTT assay revealed that transfection with the CrkL plasmid (pCMV6 CrkL) upregulated cell proliferation in the two cell lines compared with the control group. (B) A Matrigel invasion assay revealed that CrkL transfection increased the number of invading cells. (C) An MTT assay revealed that CrkL transfection enhanced cell viability in HeLa and CaSki cells treated with cisplatin compared with the control group. * $\mathrm{P}<0.05$. CrkL, Crk-like; MTT, methyl thiazolyl tetrazolium; pCMV6 EV, empty vector. 
A

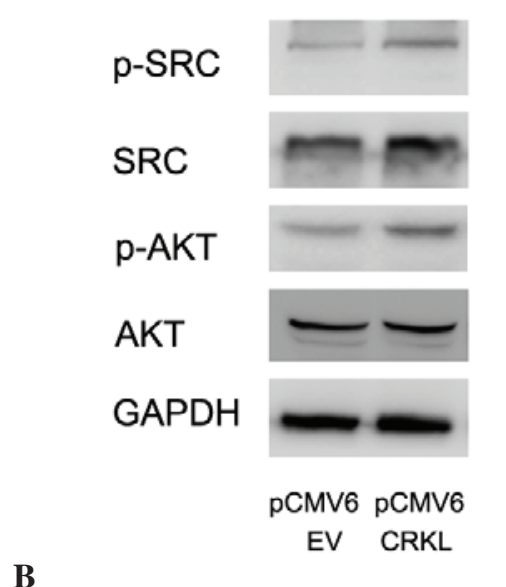

B

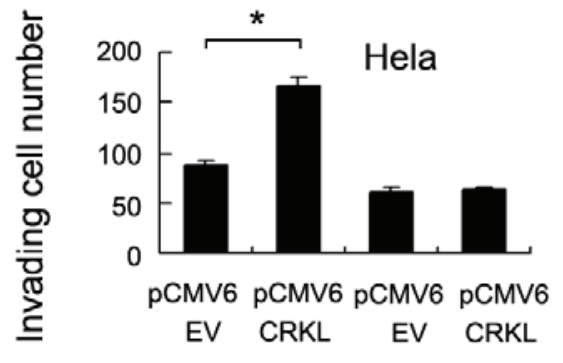

dasatinib $(0.1 \mu \mathrm{M})$
Caski

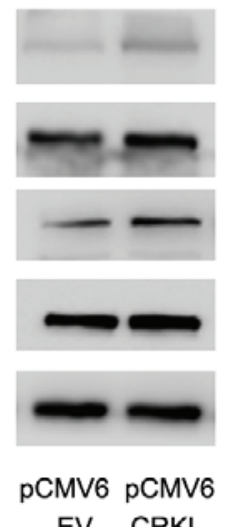

EV CRKL

Figure 4. CrkL promotes cervical cancer cell invasion through Src signaling pathways. (A) Western blot analysis revealed that transfection with the CrkL plasmid (pCMV6 CrkL) significantly upregulated the level of p-Src and p-Akt, without significant changes in the total amounts of Src and Akt. (B) The Src inhibitor dasatinib $(0.1 \mu \mathrm{M}, 3 \mathrm{~h})$ blocked the effects of CrkL on cancer cell invasion (Hela, ${ }^{*} \mathrm{P}=0.003$, CRKL plasmid vs. CRKL plasmid + dasatinib; Caski, " $\mathrm{P}=0.006$, CRKL plasmid vs. CRKL plasmid + dasatinib). CrkL, Crk-like; p-, phosphorylated; GAPDH, glyceraldehyde 3-phosphate dehydrogenase; pCMV6 EV, empty vector.

was solubilized in $150 \mu \mathrm{l}$ of DMSO. Culture medium without cells was used as the control. Each solution was measured spectrophotometrically at $490 \mathrm{~nm}$.

Cell invasion assay. A cell invasion assay was performed using a Costar 24-well Transwell chamber with a pore size of $8 \mu \mathrm{m}$. The inserts were coated with $20 \mu 1$ Matrigel (1:4dilution; BD Biosciences, San Jose, CA, USA). At $48 \mathrm{~h}$ after the transfection, cells were trypsinized and $3 \times 10^{5}$ cells in $100 \mu \mathrm{l}$ of serum-free medium were transferred to the upper Matrigel-coated chamber and incubated for $16 \mathrm{~h}$; the lower chamber contained medium supplemented with $10 \%$ FCS as the chemoattractant. Following incubation, the non-invaded cells on the upper membrane surface were removed with a cotton tip, and the cells that passed through the filter were fixed with $4 \%$ paraformaldehyde and stained with hematoxylin. The number of invaded cells was counted in 10 randomly selected high-power fields under a microscope (BX53; Olympus Corporation, Tokyo, Japan). This experiment was performed in triplicate.

Statistical analysis. SPSS software version 11.5 for Windows (SPSS, Inc., Chicago, IL, USA) was used for all statistical analyses. A $\chi^{2}$ test was used to examine the possible correlations between CrkL expression and clinicopathological factors. A Student's $t$-test was used to compare densitometry data between control and CrkL-transfected cells. All P-values are based on a two-sided statistical analysis, and $\mathrm{P}<0.05$ was considered to indicate statistical significance.

\section{Results}

Expression of CrkL in human cervical carcinoma. CrkLexpression was located in the nucleus or cytoplasm of cancer cells as brown staining, but could not be easily detected in normal cervical tissues (Fig. 1A). Of the 92 cervical cancer tissues, 45 (48.9\%) exhibited positive CrkL staining (Fig. 1B-D). The association of CrkL overexpression with clinicopathological characteristics was analyzed (Table I). The results indicate that positive CrkL immunostaining in cervical carcinoma was significantly associated with advanced TNM stage (stage II+III vs. stage $\mathrm{I}, \mathrm{P}=0.0165$ ) and lymph node metastasis (negative vs. positive, $\mathrm{P}=0.0212)$. No significant association was identified between CrkL level and other parameters, including age $(<50$ vs. $\geq 50$ years, $\mathrm{P}=0.2106$ ), histological type (squamous cell carcinoma vs. adenocarcinoma, $\mathrm{P}=0.9419)$ and differentiation (well/moderate vs. poor, $\mathrm{P}=0.5647$ ).

CrkL promotes cervical carcinoma cell growth, invasion and chemoresistance. To determine the effects of $\mathrm{CrkL}$ on cervical 
cancer cell lines, HeLa and CaSki cells were transfected with plasmids containing CrkL. As shown in Fig. 2, CrkL transfection increased its protein and mRNA levels in the two cell lines. An MTT assay revealed that CrkL upregulation increased the rate of cell growth [Day 5, pCMV6 EV vs. CrkL: HeLa, $0.993 \pm 0.009$ vs. $1.496 \pm 0.026$ ( $\mathrm{P}=0.002)$; CaSki, $1.126 \pm 0.028$ vs. $1.527 \pm 0.038(\mathrm{P}=0.007)]$ (Fig. 3A).

A Matrigel invasion assay was also conducted to assess the role of CrkL in cell invasion. As shown in Fig. 3B, CrkL transfection significantly increased the invasion ability of HeLa and CaSki cells [pCMV6 EV vs. CrkL: HeLa, $83 \pm 7$ vs. $151 \pm 8$ ( $\mathrm{P}=0.013)$; $\mathrm{CaSki}, 65 \pm 3$ vs. $132 \pm 11$ ( $\mathrm{P}=0.014)$ ] (Fig. $3 \mathrm{~B})$.

In order to investigate the role of CrkL on the chemoresistance of cervical carcinoma cells, control cells and CrkL-transfected cells were treated with cisplatin $(10 \mu \mathrm{M})$ followed by analysis of cell viability using MTT. Compared with the control group, CrkL transfection significantly increased cell viability following $48 \mathrm{~h}$ of cisplatin treatment (Fig. $3 \mathrm{C}$ ), suggesting that CrkL is important in chemoresistance of cervical cancer cells.

CrkL promotes cell invasion through Src-dependent pathways. In order to investigate the molecular pathways underlying CrkL-induced cell growth and invasion, several signaling pathways were examined. The levels of Src and Akt phosphorylation were significantly increased following CrkL transfection (Fig. 4A).

Src activation has been reported to be associated with cancer cell invasion (23). To validate its involvement in CrkL-induced cervical cancer invasion, the Src inhibitor dasatinib $(0.1 \mu \mathrm{M}$, $3 \mathrm{~h})$ was employed to treat CrkL-transfected and control cells. As shown in Fig. 4B, dasatinib treatment eliminated the effect of CrkL on cell invasion in HeLa and CaSki cell lines (Fig. 4B).

\section{Discussion}

The expression and biological functions of CrkL have been implicated in numerous types of human malignancy, including breast, lung, pancreatic and colorectal carcinomas $(17,18,21,24)$. However, the involvement of CrkL in human cervical carcinoma has not been reported. The present study examined CrkL protein expression in 92 cases of cervical carcinoma and found that CrkL was overexpressed in $48.9 \%$. Statistical analysis indicated that $\mathrm{CrkL}$ overexpression was correlated with advanced TNM grade and lymph node metastasis, suggesting its association with cervical cancer invasion. The present study revealed that $\mathrm{CrkL}$ is overexpressed in human cervical carcinoma and is associated with an advanced stage of disease, which is in accordance with previous data that confirmed CrkL as an oncogene (24-26).

CrkL contains $\mathrm{SH} 2$ and $\mathrm{SH} 3$ domains that mediate protein-protein interactions and regulate diverse cellular processes $(21,27-29)$. Several studies have suggested that CrkL may be involved in the progression of solid tumors by regulating cell proliferation and invasion (28-30). In the current study, $\mathrm{HeLa}$ and CaSki cells were transfected with a CrkL plasmid and the effects on cell proliferation and invasion were examined. In support of the immunohistochemical results, CrkL transfection significantly upregulated the rate of cell growth and invasion ability in of the two cell lines.

To the best of our knowledge, the association of CrkL and chemoresistance has not been reported previously. In the current study, CrkL transfection was found to increase cervical cancer cell viability following cisplatin treatment, which suggests that CrkL may function as an important modifier of chemoresistance in cervical carcinoma cells.

The potential mechanism of CrkL in cervical cancer cell invasion was also explored. Previous reports have indicated that CrkL functions as an adaptor protein that links Src and C3G proteins $(31,32)$. Increased activity of $\mathrm{Src}$ is a frequent occurrence in many types of human cancer, and there is growing evidence of a prominent role of Src in invasion and in other tumor progression-related events, such as the epithelial-mesenchymal transition and development of metastasis (33-35). Thus, the present study assessed the level of Src phosphorylation in CrkL-overexpressing HeLa and CaSki cell lines, and determined that CrkL transfection significantly upregulated Src phosphorylation, suggesting that Src may be involved in CrkL-induced cell invasion. To further validate this, the Src inhibitor dasatinib was employed. Dasatinib treatment significantly downregulated the invasion ability of cervical cancer cells and eliminated the invasion-promoting function of CrkL. These results demonstrate that CrkL may promote cervical cancer cell invasion through activation of Src signaling pathways. In addition, CrkL was also found to promote Akt phosphorylation; Akt signaling activation has been reported to be involved in the chemoresistance of cancer cells through antiapoptotic proteins such as Bcl-2 and Bcl-xL $(36,37)$. Thus it is possible that CrkL increases cervical cancer chemoresistance through activating Akt signaling.

In conclusion, $\mathrm{CrkL}$ is overexpressed in human cervical carcinomas and is associated with advanced stage and nodal metastasis. CrkL may also increase cervical cancer cell proliferation and chemoresistance, and promote tumor invasion through activation of Src signaling. Thus, CrkL may potentially serve as a novel therapeutic target for cervical carcinoma.

\section{References}

1. Siegel R, Ma J, Zou Z and Jemal A: Cancer statistics, 2014. CA Cancer J Clin 64: 9-29, 2014.

2. Wang T, Wu MH, Wu YM and Zhang WY: A population-based study of invasive cervical cancer patients in Beijing: 1993-2008. Chin Med J (Engl) 128: 3298-3304, 2015.

3. Angioli R, Plotti F, Luvero D, Aloisi A, Guzzo F, Capriglione S, Terranova C, De Cicco Nardone C and Benedetti-Panici P: Feasibility and safety of carboplatin plus paclitaxel as neoadjuvant chemotherapy for locally advanced cervical cancer: A pilot study. Tumour Biol 35: 2741-2746, 2014.

4. Organista-Nava J, Gómez-Gómez Y and Gariglio P: Embryonic stem cell-specific signature in cervical cancer. Tumour Biol 35: 1727-1738, 2014.

5. Du PL, Wu KS, Fang JY, Zeng Y, Xu ZX, Tang WR, Xu XL and Lin K: Cervical cancer mortality trends in China, 1991-2013, and predictions for the future. Asian Pac J Cancer Prev 16: 6391-6396, 2015.

6. Wentzensen N, Schwartz L, Zuna RE, Smith K, Mathews C, Gold MA, Allen RA, Zhang R, Dunn ST, Walker JL and Schiffman M: Performance of p16/Ki-67 immunostaining to detect cervical cancer precursors in a colposcopy referral population. Clin Cancer Res 18: 4154-4162, 2012.

7. Schwarz JK, Payton JE, Rashmi R, Xiang T, Jia Y, Huettner P, Rogers BE, Yang Q, Watson M, Rader JS and Grigsby PW: Pathway-specific analysis of gene expression data identifies the PI3K/Akt pathway as a novel therapeutic target in cervical cancer. Clin Cancer Res 18: 1464-1471, 2012. 
8. Rhodes J, York RD, Tara D, Tajinda K and Druker BJ: CrkL functions as a nuclear adaptor and transcriptional activator in Bcr-Abl-expressing cells. Exp Hematol 28: 305-310, 2000.

9. Feller SM: Crk family adaptors-signalling complex formation and biological roles. Oncogene 20: 6348-6371, 2001.

10. Fidler IJ and Kripke ML: Genomic analysis of primary tumors does not address the prevalence of metastatic cells in the population. Nat Genet 34: 23; author reply 25, 2003.

11. ten Hoeve J, Morris C, Heisterkamp N and Groffen J: Isolation and chromosomal localization of CRKL, a human crk-like gene. Oncogene 8: 2469-2474, 1993.

12. Sattler M, Salgia R, Shrikhande G, Verma S, Pisick E, Prasad KV and Griffin JD: Steel factor induces tyrosine phosphorylation of CRKL and binding of CRKL to a complex containing c-kit, phosphatidylinositol 3-kinase, and p120 (CBL). J Biol Chem 272: 10248-10253, 1997.

13. Oda T, Heaney C, Hagopian JR, Okuda K, Griffin JD and Druker BJ: Crkl is the major tyrosine-phosphorylated protein in neutrophils from patients with chronic myelogenous leukemia. J Biol Chem 269: 22925-22928, 1994.

14. Beroukhim R, Mermel CH, Porter D, Wei G, Raychaudhuri S, Donovan J, Barretina J, Boehm JS, Dobson J, Urashima M, et al: The landscape of somatic copy-number alteration across human cancers. Nature 463: 899-905, 2010.

15. Senechal K, Halpern J and Sawyers CL: The CRKL adaptor protein transforms fibroblasts and functions in transformation by the BCR-ABL oncogene. J Biol Chem 271: 23255-23261, 1996.

16. van 't Veer LJ, Dai H, van de Vijver MJ, He YD, Hart AA, Mao M, Peterse HL, van der Kooy K, Marton MJ, Witteveen AT, et al: Gene expression profiling predicts clinical outcome of breast cancer. Nature 415: 530-536, 2002

17. Lin F, Chengyao X, Qingchang L, Qianze D, Enhua W and Yan W: CRKL promotes lung cancer cell invasion through ERK-MMPO pathway. Mol Carcinog 54 (Suppl 1): E35-E44, 2015.

18. Wang Y, Dong QZ, Fu L, Stoecker M, Wang E and Wang EH: Overexpression of CRKL correlates with poor prognosis and cell proliferation in non-small cell lung cancer. Mol Carcinog 52 890-899, 2013.

19. Senechal K, Heaney C, Druker B and Sawyers CL: Structural requirements for function of the $\mathrm{Crkl}$ adapter protein in fibroblasts and hematopoietic cells. Mol Cell Biol 18: 5082-5090, 1998.

20. Schönherr C, Yang HL, Vigny M, Palmer RH and Hallberg B: Anaplastic lymphoma kinase activates the small GTPase Rapl via the Rap1-specific GEF C3G in both neuroblastoma and PC12 cells. Oncogene 29: 2817-2830, 2010.

21. Zhao T, Miao Z, Wang Z, Xu Y, Wu J, Liu X, You Y and Li J: Overexpression of CRKL correlates with malignant cell proliferation in breast cancer. Tumour Biol 34: 2891-2897, 2013.

22. Livak KJ and Schmittgen TD: Analysis of relative gene expression data using real-time quantitative PCR and the 2(-Delta Delta C(T)) Method. Methods 25: 402-408, 2001.

23. Zhao S, Li H, Wang Q, Su C, Wang G, Song H, Zhao L, Luan Z and Su R: The role of c-Src in the invasion and metastasis of hepatocellular carcinoma cells induced by association of cell surface GRP78 with activated $\alpha 2 \mathrm{M}$. BMC Cancer 15: 389, 2015.
24. Tamura M, Sasaki Y, Kobashi K, Takeda K1, Nakagaki T, Idogawa $\mathrm{M}$ and Tokino T: CRKL oncogene is downregulated by p53 through miR-200s. Cancer Sci 106: 1033-1040, 2015.

25. Ladanyi M: CRKL as a lung cancer oncogene and mediator of acquired resistance to EGFR inhibitors: Is it all that it is cracked up to be? Cancer Discov 1: 560-561, 2011.

26. Koval AP, Karas M, Zick Y and LeRoith D: Interplay of the proto-oncogene proteins CrkL and CrkII in insulin-like growth factor-I receptor-mediated signal transduction. J Biol Chem 273: 14780-14787, 1998.

27. Fu L, Dong Q, Xie C, Wang Y and Li Q: CRKL protein overexpression enhances cell proliferation and invasion in pancreatic cancer. Tumour Biol 36: 1015-1022, 2015.

28. Singer CF, Hudelist G, Lamm W, Mueller R, Handl C, Kubista E and Czerwenka K: Active (p)CrkL is overexpressed in human malignancies: Potential role as a surrogate parameter for therapeutic tyrosine kinase inhibition. Oncol Rep 15: 353-359, 2006.

29. Kim YH, Kwei KA, Girard L, Salari K, Kao J, Pacyna-Gengelbach M, Wang P, Hernandez-Boussard T, Gazdar AF, Petersen I, et al: Genomic and functional analysis identifies CRKL as an oncogene amplified in lung cancer. Oncogene 29: 1421-1430, 2010.

30. Fathers KE, Bell ES, Rajadurai CV, Cory S, Zhao H, Mourskaia A, Zuo D, Madore J, Monast A, Mes-Masson AM, et al: Crk adaptor proteins act as key signaling integrators for breast tumorigenesis. Breast Cancer Res 14: R74, 2012.

31. Graf R, Barbero S, Keller N, Chen L, Uryu S, Schlaepfer D and Stupack D: Src-inducible association of CrkL with procaspase- 8 promotes cell migration. Cell Adh Migr 7: 362-369, 2013.

32. Shen Q, Rahn JJ, Zhang J, Gunasekera N, Sun X, Shaw AR, Hendzel MJ, Hoffman P, Bernier A and Hugh JC: MUC1 initiates Src-CrkL-Rac1/Cdc42-mediated actin cytoskeletal protrusive motility after ligating intercellular adhesion molecule-1. Mol Cancer Res 6: 555-567, 2008.

33. Guarino $\mathrm{M}$ : Src signaling in cancer invasion. J Cell Physiol 223: 14-26, 2010

34. Cui A, Hua H, Shao T, Song P, Kong Q, Luo T and Jiang Y: Aflatoxin B1 induces Src phosphorylation and stimulates lung cancer cell migration. Tumour Biol 36: 6507-6513, 2015.

35. Sun B, Yang N, Jiang Y, Zhang H, Hou C, Ji C, Liu Y and Zuo P: Antagomir-1290 suppresses $\mathrm{CD} 133^{+}$cells in non-small cell lung cancer by targeting fyn-related Src family tyrosine kinase. Tumour Biol 36: 6223-6230, 2015.

36. Wang JH, Nao JF, Zhang M and He P: 20 (s)-ginsenoside Rg3 promotes apoptosis in human ovarian cancer HO-8910 cells through PI3K/Akt and XIAP pathways. Tumour Biol 35: 11985-11994, 2014

37. Ye G, Lu Q, Zhao W, Du D, Jin L and Liu Y: Fucoxanthin induces apoptosis in human cervical cancer cell line HeLa via PI3K/Akt pathway. Tumour Biol 35: 11261-11267, 2014. 